\title{
Comprehensive Real-Time Bridge Health Monitoring System of Tongtai Bridge
}

\author{
Su-su LEI ${ }^{1}$, Yong-tao GAO ${ }^{1}$, Dan-guang PAN ${ }^{1, a}$ \\ ${ }^{1}$ School of Civil and Environmental Engineering, University of Science and Technology Beijing, Beijing 100083, China
}

\begin{abstract}
Tongtai Bridge is the world's largest suspension curve-girder-skew-arch bridge, which is located in Zhangjiakou, China. The understanding of mechanics characteristics is limited to such complex bridges, so it is necessary to establish reliable health monitoring system to investigate the static and dynamic responses and monitor the safety of the bridge. A comprehensive real-time bridge health monitoring system is establish, which includes four aspects: sensor system, data acquisition and transmission system, data processing and control system, structure early warning and security assessment system. The paper systematically describes the system design principles, sensor layout, and monitoring content, then expounds system integration and function of each subsystem.
\end{abstract}

\section{INTRODUCTION}

After the bridge was opened to traffic, the material aging subjects to weather, corrosion.etc, fatigue occurs under long term dynamic loads, as well as damage appears due to hit by a car, overloaded vehicles, strong winds and other uncertainties is inevitable. Especially, with the design of long-span bridges is softer, the structural form and function is increasingly complex in recent years, in order to insure the carrying capacity, operation status, safety and durability of the bridge during the operation, establish a bridge structural health monitoring system is must[1-5].

Traditional bridge safety assessment was from visual inspection or measured information by artificial portable instruments, which has the disadvantages of long cycle, poor timeliness, localized detection, so the traditional method of manual bridge inspection has significant limitations in practical application[6,7]. Bridge structural monitoring system combines modern sensor technology, network communication technology, signal processing and analysis techniques, data management, forecasting techniques and structural analysis theory, greatly extends the bridge monitoring field, improve the reliability of prediction evaluation[8].

\section{System Design}

Zhangjiakou Tongtai Bridge is the world's largest curved girder skew through suspension arch bridge. The arch spans diagonally over the main bridge girder, moreover the angle between the horizontal projection of the arch and the tangential of main girder axis is $19.5^{\circ}$. Oblique arches, curved girder and diagonal cross cable mesh constitutes a unique space form of the bridge

\footnotetext{
a Corresponding author: pdg@ustb.edu.cn
}

structure. The main span is a curved steel box girder with $190 \mathrm{~m}$ length, the bending radius is $600 \mathrm{~m}$. The arch maximum rise is $62.118 \mathrm{~m}$, arch spacing is $180 \mathrm{~m}$, risespan ratio is 0.3451 . The girder is a flat steel box girder, with height $3 \mathrm{~m}$. The arch is a single cell box cross-section, width $7.04 \mathrm{~m}$, height $3.8 \mathrm{~m}$. Bridges photo, girder box boxsection and arch box-section are shown in Figure 1.

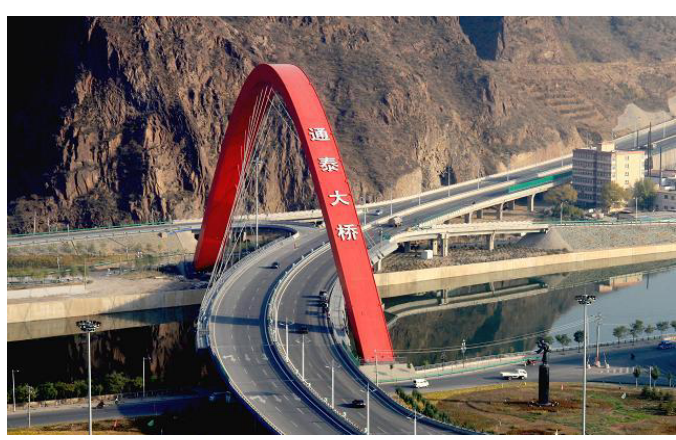

(a) Bridges photo

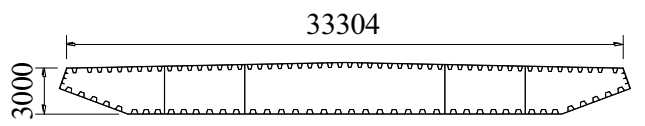

(b) box girder cross-section (mm) 


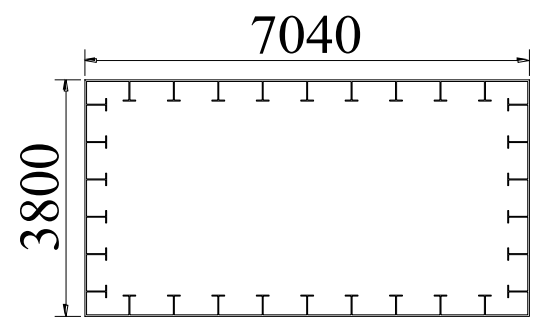

(c) box arch cross-section (mm)

Fig.1 The pictures of the bridge

Tongtai Bridge is official opened to traffic in November 2008, and it has been operating for six years. The weakening performance of the bridge is a long and lengthy process. Their own state is gradual degradation, then the corresponding inspection, repair and reinforcement work will be gradually increased. On the current development, the advanced monitoring technology and traditional detection methods are two complementary aspects of bridge management. There is less maintenance work during the early use of the bridge, focusing on observation and information measured by monitoring systems, and establishing accurate and reliable prediction model in order to prepare long-term stability inspection and maintenance plan. In the entire design life of the bridge, based on the premise of the bridge safe and reliable operation, using automatic monitoring and security warning system, makes the maintenance and management of the bridge holding at a relatively steady level. In addition, the establishment of a technologically advanced, stable and efficient structural monitoring system is of great importance for enhancing the design, construction and management of bridge engineering.

Zhangjiakou is belong to temperate continental monsoon climate, with perennial gale; located in the junction of Beijing, Hebei, Shanxi and Inner Mongolia, which is an important transportation hub; and located in the North China Plain seismic belt, in which place a 6.2 magnitude Zhangbei earthquake occurred in 1998 and a 4.3 magnitude earthquake occurred in September this year. To ensure the safe and economical operation of Tongtai Bridge, economic rationality maintenance in the service, and make timely safety assessment after the occasional unexpected factors occurs, a comprehensive real-time automatic monitoring and evaluation system of the bridge will establish. The main objectives of the system design are:

(1) Make safety evaluation of the bridge under sudden load as strong winds, earthquake and hit by a car, etc.

(2) Monitoring the stress distribution and settlement;

(3) Making safety assessment of the structure under the current situation;

(4) Provide a theoretical basis and the appropriate conservation plans for bridge maintenance.

When the structural damage occurs, some of the local and global response of the structure will exhibit different characteristics with normal state, through the installation of the sensor system is able to obtain such information, and the changes of this information before and after damage can identify the location and relative degree of these damage. Through long-term observation and analysis of the damage sensitive feature, can master the evolution of bridge performance degradation, in order to deploy the appropriate improvement measures, to extend the service life of the bridge.

\section{Monitoring Content and Means}

Bridges automatic monitoring system covers all main load bearing components. In order to achieve real-time safety and scientific evaluation of the bridge, according to the structural characteristics of Tongtai Bridge, the system needs to obtain information about the data used as a scientific basis. In addition, seismic response monitoring is added to this system, so that the bridge can still be effectively monitored in extreme cases.

The monitoring system includes the following four areas: (1) Environmental monitoring: wind loads (including wind speed, wind direction), temperature and humidity (including air temperature and humidity, the structure temperature field); (2) The static characteristics monitoring: static strain of the arch and the main girder, the bridge space displacement (including arch, bearing and girder spatial displacement b); (3) The dynamic characteristics monitoring: dynamic strain of the main beam, the vibration of the arch and the main girder, seismic response, cable tension and vibration; (4) Other monitoring: vehicle weighing system, the main girder bearing displacement. Sensor layout is shown in Figure 2

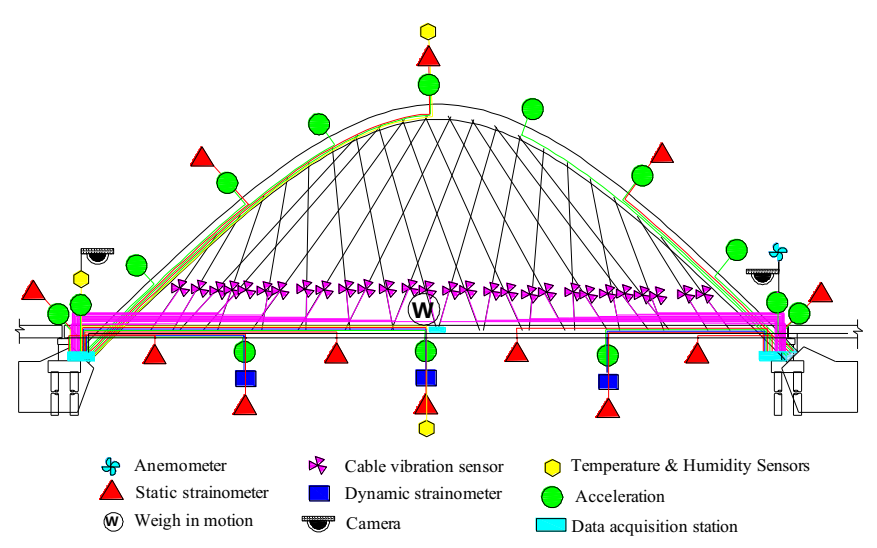

Fig.2 Sensor layout

Wind Load. Wind load monitoring includes wind speed and direction data. For long-span bridges, consider the role of wind load is essential. Under lateral wind excitations, the main girder and arch will produce lateral displacement. Continuously collecting wind speed and direction information of wind loads provide data for the analysis of bridges real-time situation, so wind load monitoring is an important part of the automatic monitoring system.

Temperature and Humidity. Through monitoring the distribution of temperature field of bridge, providing original data for numerical analysis of temperature effect, making comparison and quantitative analysis for the changes of bridge operation state at different temperatures status, such as bridges deformation, stress changes, etc. In the automatic monitoring system, the 
temperature sensors are connected with the network nodes in parallel, communicating with the computer through a network bus, to achieve automatic remote monitoring temperature.

Arch and Girder Static Strain. The purpose of monitoring arch and girder static strain is that researching the internal force distribution of the structure, the local structure and joint in response to various loads, in order to provide the basis for structure damage identification, fatigue damage assessment and structural life assessments. In this system, the number of the static strain measurement points is 92 , including 52 in the main girder and 40 in the arch rib.

Spatial Displacement. Monitoring the deformation of arch axis, bearing settlement and the main girder spatial displacement, and painting the corresponding displacement deformation influence line and surface in order to detect the spatial deformation state of the control parts, provide basis for an overall assessment of the carrying capacity, the operating status and the durable ability of the bridge. It is intended to use conventional measurement methods to measure space displacement of the bridge, arranging measuring point in key positions of the bridge, measuring the respective point every one month, then analysis the force status of the bridges by data collection.

Girder Dynamic Strain. Dynamic strain generated under live loads on the bridge, generally higher than the corresponding static strain generated by the same static load. The ratio of dynamic strain to corresponding strain is called the live load impact factor, which reflects the dynamic effect of dynamic loads to the bridge. Measured value of impact factor reflects the driving performance of the bridge, while the factor is large means the driving performance is poor, and the bridge flatness is poor, vice versa. Live load impact factor relate to bridge structure forms, vehicle speed, bridges flatness and other factors. For real-time monitoring the impact factor of the bridge structure, giving a true reflection of the actual stress state of the bridge, carrying out the main girder dynamic strain monitoring is essential.

Arch and Main Girder Vibration. The change of the dynamic performance of the bridge reflects the change of the stiffness properties of the bridge, thus getting the dynamic characteristics that is also a "fingerprint" of the structure Through monitoring the vibration of the arch and girders, not only can identify the dynamic characteristics of the bridge structure, but also on the record the time history of the girder under fluctuating load. There are 45 unidirectional accelerometers arranged for structural vibration monitoring in the system, which constitute 15 tri-axial accelerometers.

Earthquake Response. In the event of earthquake, the bridge vibrates, so that the structure generates displacement, velocity, acceleration, internal force and deformation response, which is change over time. In order to obtain the internal force and deformation of the bridge under seismic loads, seismic response data is needed. The system uses a tri-axial force balance accelerometers to measure seismic response, which can simultaneously measure the north-south direction, eastwest direction and vertically vibration, can accurately measure a variety of low-frequency vibration signals and the pulse signal of the earth as well.

Cable Force and Vibration. Cable is an important part of the cable-stay bridge. According to the needs of the cable force automatic long-term monitoring, the project uses the indirect method to measure cable force by the vibration frequency. The system is arranged 28 unidirectional accelerometers, one accelerometer each cable.

Weigh in Motion. Vehicle load is an important part of bridge load, accessing accurate and real-time vehicle load data effectively guaranteed the normal operation of bridge automatic monitoring system. In order to monitor vehicle traffic conditions, camera devices are arranged in each lane of the bridge.

Girder Bearing Displacement. According to Tongtai Bridge operational requirements, displacement sensor is installed to the large-scale girder bearings for real-time monitoring, in order to grasp the real-time operational status of the bridge. Bearings transfer the load from the main girder, bearing displacement reflects the changes of the main girder, 12 unidirectional displacement sensors are arranged, each one in longitudinal, transverse and vertical direction.

\section{System Integration}

The entire system health monitoring is consists of sensors system, data acquisition and transmission systems, data processing and control systems, structural safety warning assessment system four subsystem, shown in Figure 3.

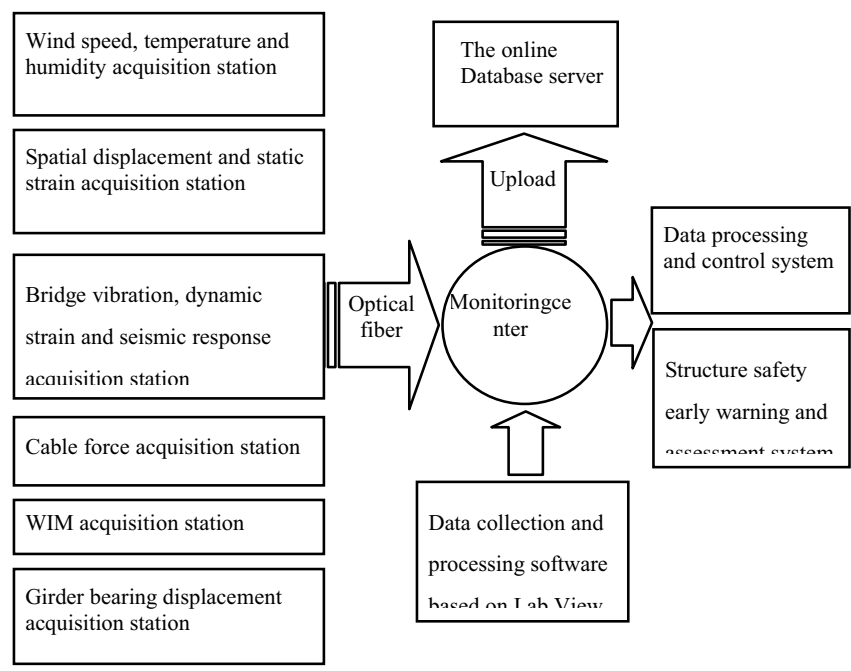

Fig.3 System integration

Data Acquisition and Transmission System. All data collected by sensors is transmitted to monitoring center through the optical fibres. Equipment installed in monitoring center includes: standard cabinets, servers, workstations, switches.

Data Processing and Control System. Through data processing software which is developed based on Lab View and systems database which is developed based on Oracle, the data collected in the field is processed and 
uploaded to server, in order to implement remote check and control functions.

Structural Early Warning and Security Assessment System. The ultimate goal of bridge health monitoring is establish a early warning and security assessment system, setting warning threshold through finite element analysis and data analysis results, warning in the abnormal state. In the design of online health monitoring system, paying attention to more detailed offline analysis is also required.

\section{Conclusions}

This paper describes the comprehensive real-time automatic bridge health monitoring system of Tongtai Bridge, which provides reference research for health monitoring system design of special bridges. The main conclusions are:

(1) Large-scale health monitoring system includes many subsystems, through data acquisition and processing software developed based on Lab View, the system supports different types of monitoring devices to access, the interface and data communications between the various health monitoring subsystem is implemented;

(2) The system enables remote network control, can freely configure function parameter type, while monitoring parameter types, monitoring data processing and display method can be modified through background configuration,;

(3) Comprehensive bridge health monitoring system can directly reflect the specific circumstances of the bridge in real-time operations, and provide the basis for the bridge daily management and maintenance;

(4) According to the monitoring data of large-scale structure, inversing its structural health status and possible structural damage, is inverse problem of a complex structure system under imperfect conditions, thus the structure status inversion, damage detection and condition assessment still need in-depth study.

\section{Acknowledgements}

This study was supported by the Beijing Natural Science Foundation of China through Grants 8143037. These supports are gratefully acknowledged.

\section{References}

1. T. Y. Liu, W. L. Chiang, C. W. Chen, et al. Identification and monitoring of bridge health from ambient vibration data [J]. Journal of Vibration and Control, 17.4 (2011): 589-603.

2. M Liu, M. F. Dan, and S.Y Kim. Bridge system performance assessment from structural health monitoring: a case study [J]. Journal of Structural Engineering 135.6 (2009): 733-742.

3. F. Magalhães, A. Cunha, and E. Caetano. Vibration based structural health monitoring of an arch bridge: from automated OMA to damage detection [J]. Mechanical Systems and Signal Processing 28 (2012): 212-228.
4. J. M. Ko, Y. Q. Ni, H. F. Zhou, et al. Investigation concerning structural health monitoring of an instrumented cable-stayed bridge [J].Structures \& Infrastructure Engineering5.6 (2009): 497-513.

5. J. P. Ou, and H Li. Structural health monitoring in mainland China: review and future trends $[\mathrm{J}]$. Structural Health Monitoring 9.3 (2010): 219-231.

6. D. Pines, A. E. Aktan. Status of structural health monitoring of long - span bridges in the United States [J]. Progress in Structural Engineering and materials 4.4 (2002): 372-380.

7. C. R. Farrar, K. Worden. An introduction to structural health monitoring [J]. Philosophical Transactions of the Royal Society A: Mathematical, Physical and Engineering Sciences 365.1851 (2007): 303-315.

8. J. P. Ou. Practical implementations of intelligent health monitoring systems in HIT [C] // Proc. of North American Euro Pacific Workshop for Sensing Issues in Civil Structural Health Monitoring. Hawaii, USA: 2004 